\title{
Addressing the Medical and Support Service Needs of People Living with HIV (PLWH) through Program Collaboration and Service Integration (PCSI)
}

\author{
Stephanie Bernard, Amrita Tailor, Patricia Jones, and Donna E. Alexander \\ Division of HIV/AIDS Prevention at the Centers for Disease Control and Prevention
}

\begin{abstract}
Background: Approximately 1.2 million Americans are living with HIV, and about 50,000 new infections occur each year. People living with HIV (PLWH) have numerous medical and psychosocial needs that impact HIV disease progression and challenge treatment outcomes. Purpose: Using CDC's Program Collaboration and Service Integration (PCSI) framework, we examined strategies, challenges, and lessons learned from a local health department's efforts to institute PCSI to address the diverse needs of their patients with HIV. Methods: We captured case study data through: 1) semi-structured interviews with key program administrators, 2) analysis of program documents, and 3) site observations and review of clinic procedures. Results: Findings highlight the importance of co-locating services, partnering to leverage resources, and conducting cross-training of staff. Providing co-located services reduced wait times and enhanced coordination of care. Partnering to leverage resources increased patient referrals and enhanced access to comprehensive services. Staff cross-training resulted in more coordinated care and efficient service delivery. Conclusion: The results show that PCSI is essential for optimal care for PLWH. Incorporating PCSI was a vital component of the health department's comprehensive approach to addressing the multiple medical and support service needs of its HIV-infected clients.
\end{abstract}

(c) 2016 Californian Journal of Health Promotion. All rights reserved.

Keywords: HIV prevention, service integration, wrap-around services, ancillary services, support services, case study

\section{Introduction}

HIV-infected persons are faced with numerous medical and psychosocial concerns that present challenges to treating their HIV infection. With improved access to antiretroviral treatment that has prolonged the lives of HIV-infected persons, HIV is increasingly a chronic condition that can be effectively managed through safer behavior choices, strict adherence to treatment, and appropriate monitoring of HIV infection and other medical and social conditions (Gallant et al., 2011). As treatment prolongs life, HIVinfected persons are faced with many of the same conditions that the general public experiences as it ages, such as heart disease and diabetes. Additionally, co-morbid conditions such as substance abuse and mental illness affect many HIV-infected persons across their life span, and can be further exacerbated by social conditions such as poverty and lack of housing
(Aidala et al., 2015; Leserman, 2008; Riley et al., 2007).

\section{Psychosocial Factors}

Mental health, substance abuse, and other psychosocial factors can play a substantial role in the HIV care and treatment outcomes of PLWH ((Aidala et al., 2015; Zahari et al., 2010; Zaller et al. 2007; Altice et al., 2010; CDC, 2012). Numerous studies document the association between psychosocial characteristics and HIV disease progression (Cole, 2008; Ironson \& Hayward, 2008; Ickovics et al., 2006). Psychosocial factors such as stress, trauma, and depression have been linked to declines in CD4 cell count, increases in viral load, and mortality among HIV-infected persons (Lee et. al., 2015; Altice et al., 2010). Studies evaluating receipt of mental health and substance abuse treatment services have shown increased entry into primary care, as well as improved clinical retention for HIV-positive 
patients (White et al., 2014; Lee et al., 2015). An integrated approach to addressing the multiple needs of PLWH, particularly mental health and substance abuse needs, should be a major component of comprehensive HIV care and treatment services (White et al., 2014; CDC, 2012).

\section{Stable Housing}

Housing instability has been linked to reduced treatment effectiveness, impacting medication access and adherence, sustained viral suppression, and engagement in risk behaviors for HIV transmission (Aidala et al., 2015; Riley et al., 2007). It is estimated that approximately half of all PLWH will need some type of housing assistance over the course of their infection (Aidala et al., 2007; Shubert and Bernstine, 2007). Systematic reviews have found that housing stability is positively associated with increased HIV medication adherence, utilization of health and social services, improved health status, and decreased HIV risk behaviors (Leaver et al., 2007). Receipt of housing assistance has also been shown to be predictive of linkage and retention in care (Aidala et al., 2015; Aidala et al., 2007). Studies support case management, housing, and transportation resources, which have been associated with enhanced linkage and retention in HIV primary care by facilitating initiation of antiretroviral therapy (ART) and improved medication adherence (Aidala et al., 2015; Evans, 2006).

\section{HIV as a Chronic Disease}

With an increasing focus on HIV as a chronic disease, strategies such as self-care and chronic disease management present potential benefits for PLWH (Swendeman et al., 2009). Studies particularly note the importance of exercise and proper nutrition for PLWH (Carter et al., 2015; O’Brien et al., 2010). In studies assessing the benefits of aerobic exercise, findings show potential increases in CD4 count, enhanced cardiopulmonary health, and heightened psychological well-being (O’Brien et al., 2010; Nixon et al., 2005). Studies also encourage adequate dietary intake of micronutrients (i.e., vitamin D3, zinc, copper and selenium) for PLWH, citing reduced risk of HIV disease progression, morbidity and mortality (Mahlungulu S. et al., 2007; Carter et al., 2015). Additional research is needed to further examine the effect of such strategies on treatment outcomes.

\section{Program Collaboration and Service Integration}

Program collaboration and service integration (PCSI) is a framework for organizing and blending interrelated health issues, activities, and prevention strategies to facilitate a more comprehensive delivery of services (CDC, 2012). A recent Institute of Medicine (IOM) report asserts that a "comprehensive, multidisciplinary approach is needed for HIV/AIDS services due to the complexity of issues implicated in the health of individuals with HIV/AIDS”(IOM, 2011). Over the years, there has been an increasing shift in collaboration and integration of prevention services related to HIV, other sexually transmitted diseases (STD), viral hepatitis, and tuberculosis (TB) in the United States, as these diseases are synergistically interacting epidemics, or syndemics (CDC, 2009). For PLWH or persons who are at risk of HIV acquisition, examples of these interactions include, increased risk of HIV acquisition among persons with STDs; common risks and modes of transmission for HIV, viral hepatitis and STDs; and TB as an AIDS-defining opportunistic infection which can accelerate HIV progression (CDC, 2009).

CDC defines program collaboration as a relationship between two or more entities that is mutually beneficial, and involves a sharing of resources, common goals, responsibilities and successes (CDC, 2009). Program collaboration can result in better service integration at the person level, but this is not an absolute consequence. Service integration is defined as a mechanism of service delivery that aims to provide services from multiple programs that appear seamless to clients due to reduced administrative barriers (CDC, 2009). Three levels of service integration, include: 1) nonintegrated services, where services are provided for a single condition; 2) core integrated services where two or more CDC-recommended services 
are integrated into clinical care; and 3) a comprehensive set of best and promising evidence-based practices integrated into health and social services (Spencer et al., 2013).

Integration of services is particularly important to ensure access to comprehensive care and prevention. CDC proposes multiple levels of service integration at the client-provider interface that allow jurisdictions to increase efficiency and reduce redundancy and missed opportunities for prevention and treatment. Multiple levels of service integration also increase flexibility of responses to evolving epidemics by enabling partners to adapt or modify policies, and increase control over operations by using local information derived from surveillance, program data, and key performance indicators (CDC, 2009; CDC, 2012). A transition of the HIV health care model from one that focuses solely on management of HIV infection to a more holistic approach that provides comprehensive services addressing the array of physical and psychosocial needs of individuals is consistent with the PCSI framework (Sackoff et al., 2006).

\section{Current Study}

During the process of updating CDC's "Recommendations for HIV Prevention with Adults and Adolescents with HIV in the United States," we began examining the importance of an integrated approach to addressing the multiple medical and support service needs of PLWH. Using CDC's PCSI framework, this study presents an overview of a local health department's efforts to incorporate program collaboration and service integration in their care and prevention services for PLWH. It is intended to provide lessons learned and a potential model for other health departments considering greater collaboration and/or integration of services. The specific purpose of this study is to: 1) examine the extent to which a local health department implemented PCSI in its current program to address the diverse needs of their HIV-infected clientele, 2) compare the program to CDC's PCSI model, and 3) describe some of the key strategies, challenges, and lessons learned from the implementation of PCSI.

\section{Methods}

\section{Study Design}

To examine the practical implementation of PCSI within a public health setting, we conducted a case study of a local health department funded in January 2012 under CDC's new health department program, PS 121201, "Comprehensive HIV Prevention Program for Health Departments." The Fulton County Department of Health and Wellness (FCDHW), located in the high HIV prevalence jurisdiction of Atlanta, Georgia, was the focus of this case study due to its establishment of a holistic approach to delivering services to individuals with HIV, STD, and/or TB infection. From June-July 2012, we conducted semi-structured interviews with eight key informants involved in the restructuring of communicable disease services at FCDHW. Using a convenience sample, efforts were made to include perspectives from the state, county, and federal levels. In addition to key informant interviews, a content analysis was conducted of programmatic documents used during the restructure.

\section{Study Site}

FCDHW's program illustrates a public health system responding to the diverse service needs of PLWH through enhanced integration of prevention, care, and treatment services; with a particular focus on ensuring adequate treatment for both physical and mental health conditions (Tambe et al., 2008). FCDHW is a full-service local health department that serves on average approximately 60,000 patients per year and 240 patients each day (FCDHW, 2012). FCDHW's Communicable Disease Prevention Branch currently serves primarily an African American population, with $65 \%$ male and $34 \%$ female patients (FCDHW, 2012). The majority of patients (approximately 53\%) are between 20-34 years of age. Of the HIV-infected clients served, approximately $37 \%$ have a mental health disorder and approximately $40 \%$ a substance abuse disorder, many of which have cooccurring disorders. Programmatic data from 2012 show that approximately $61 \%$ of the HIV clients are co-infected with an STD or TB infection (FCDHW, 2012). 


\section{Interview Participants}

The inclusion criteria for the key informant interviews were that participants had been involved in the conceptualization and/or implementation of plans to restructure services at FCDHW from 1999 to 2012. Key informants selected at the county level included program administrators and staff, including data managers involved in the integration of data collection and management efforts. State level participants included the PCSI coordinator and state HIV/AIDS coordinator engaged during the restructure. Also, the CDC project officer for the state of Georgia participated to offer insights from the federal level.

\section{Interview Questions}

We developed an interview protocol using CDC's PCSI framework, Essential Public Health Functions and Potential Collaboration Strategies among Programs (CDC, 2009). We asked semi-structured questions examining how PCSI was incorporated in program efforts, including: 1) How were services integrated to improve efficiencies, 2) How were community partnerships developed and mobilized to increase access to needed services, 3) What policies and procedures were put in place to ensure sustained delivery of coordinated services, and 4) What were challenges and/or facilitators to successful PCSI implementation?

\section{Procedures}

The study protocol was reviewed and approved by CDC and Fulton County officials prior to collecting data. Semi-structured interviews were conducted both in a group and individual format, over the phone and in-person at Fulton County's Aldredge Health Center and HIV primary care clinic. The first interview occurred in person and included key program administrators. At this time the initial interview protocol was used to guide the discussion. Based on responses from the first interview, additional key informants (i.e., data analyst, state contacts) were identified and asked to participate in the study. Questions for the additional interviews were built upon the initial protocol questions and responses. Due to resource constraints and scheduling challenges, some interviews were conducted via phone. Individual interviews were conducted with some staff due to the specialized nature of the questions (i.e., data collection).

Interviews were conducted by the primary author, a CDC health scientist, and two additional CDC scientists. Some questions asked over the phone were asked again in-person if further clarification was needed, which allowed interviewers to compare responses, find any discrepancies, and obtain explanations of differences. Written notes were taken throughout the interviews, and content was grouped into thematic categories, and then compared to elements in CDC's PCSI framework. Interviews were not taped so notes were not captured verbatim. Findings were reviewed and discussed by the three CDC scientists to confirm the analysis and resolve any discrepancies. Data collection methods were intended to maximize accuracy of responses.

\section{Content Analysis}

To triangulate data sources and inform the interview responses, we examined key programmatic documents used during the planning and implementation of FCDHW's restructure. The goal of this analysis was to identify evidence of programmatic efforts supporting collaboration and/or service integration. We identified 17 documents used from 1999 to 2012 showing evidence of PCSI, including: internal reports and correspondence to staff; public communication materials; guidance documents; organizational and clinic flow charts; data collection tools; and community resource lists and partner referral protocols. Documents were analyzed specifically for evidence of: 1) community partnerships and/or collaborations with service providers, and 2) changes in programmatic priorities, policies and procedures (i.e., referral protocols) facilitating greater collaboration and/or service coordination. See Table 1 for a list of documents used in the analysis. 
Table 1.

Key Documents Examined for Evidence of Program Collaboration and Service Integration, 1999-2012.

\section{Document Name}

1. Fulton County Health Services: Healthy Families, Fulton County Health Services

2. Fulton County Health Services: Healthy Communities, Fulton County Health Services

3. Interoffice Memorandum: Second Quarter Event Report, Fulton County Health Services

4. Ryan White Program: Handbook, Department of Health and Wellness, Communicable Disease Prevention Program

5. Department of Health and Wellness, Communicable Disease Prevention Branch, HIV Prevention Program Organizational Structure, 2012

6. Visual HealthNet/WEB for Public Health, Fulton County Health Services

7. Fulton County Department of Health and Wellness, Communicable Disease Prevention Branch, A Snap Shot

8. Fulton County Department of Health and Wellness, Organizational Chart, 1999

9. Fulton County Department of Health and Wellness, Sexually Transmitted Infection Clinic Flow Plan

10. Evidence-based Intervention Integration: Implementation in Healthcare Setting Flowchart

11. Sexually Transmitted Infections Clinic Resource, Fulton County Health Services

12. HIV Primary Care Resource, Fulton County Health Services

13. Tuberculosis Prevention and Treatment Program Resource, Fulton County Health Services

14. Community Resource Guide to HIV Prevention Services, Fulton County Health Services

15. Interoffice Memorandum: List of Collaborations with Substance Abuse Treatment Providers

16. Referral Protocols and Procedures Guidance, Fulton County Department of Health and Wellness

17. Fulton County Department of Health and Wellness, Communicable Disease Prevention Branch, Monthly report, June 2012
Document Type

Communication materials

Communication materials

Public

Internal communication

Guidance document

\section{Public and staff}

Organizational Chart

Public and staff

Guidance document

Staff

Guidance document

Staff

Organizational chart

Staff

Clinic flow chart

Staff

Clinic flow chart

Staff

Communication materials Public

Communication materials

Public

Communication materials

Public

Guidance document

Public

Internal communication

Staff

Guidance Document

Staff

Internal report 


\section{Interview Results}

\section{Emerging Themes}

Three overarching themes emerged from the interviews, including, the importance of:1) a holistic approach to treating HIV infected clients, 2) partnering to leverage resources, and 3) increasing efficiencies in service delivery. These factors were identified as key to ensuring HIV- infected clients received optimal care and support services.

\section{A Holistic Approach to HIV Treatment "16 years ago programs operated in silos led by three separate program administrators”. -Program administrator}

Interviews with program administrators and staff revealed that in 1999, officials within the Fulton County Department of Health (DOH) began discussions about ways to more efficiently meet the complex needs of patients with communicable diseases. Such discussions were the start of a challenging undertaking to revamp the way services were delivered to patients suffering from HIV, STD, and/or TB infection. Program administrators noted that, until that time, HIV, STD and TB services were operating in silos, with very little coordination of efforts. Patients suffering from multiple conditions were subject to duplicative intake, screening, and assessment procedures, longer waiting times to access services, and difficulty navigating services with varying funding mechanisms and programmatic structures.

To address such challenges, and to convey the importance of a holistic approach to prevention, care and treatment, program administrators reported that the Fulton County $\mathrm{DOH}$ was renamed, and the three separate HIV, STD, and TB programs that had been run by separate administrators, were combined to create the Communicable Disease Prevention Services Branch (Tambe et al., 2008). Program administrators maintained that through this new branch, comprehensive prevention, care and treatment services could be provided within and across the various TB, HIV, and STD clinics, thereby reducing redundancy and challenges navigating services.

\section{Leveraging Resources through Program Collaboration}

"We work with community-based organizations to go into non-traditional settings such as bars and clubs to provide prevention services".

-Program staff

To increase FCDHW's reach and enhance its capacity to provide comprehensive services to patients, program staff and administrators noted the establishment of numerous formal relationships with a variety of service providers, including community-based organizations (CBOs), federally qualified health centers, correctional facilities, and substance abuse and mental health care facilities. For example, program staff spoke extensively about FCDHW's ongoing partnership with the nonprofit organization, AID Atlanta, an organization which provides support services through case management, housing, and employment assistance programs. Case managers from AID Atlanta are made available onsite at FCDHW's clinics to ensure patients with support service needs are able to easily access services and to enhance coordination among services and providers. Program staff also mentioned working with substance abuse agencies throughout Atlanta to provide referrals to inpatient treatment services; while less intensive counseling was available onsite.

Additionally, program administrators reported that FCDHW partners with health centers and community-based organizations to conduct prevention activities and provide screening and treatment services in the community. According to interviewees, FCDHW's mobile van program has been a valuable asset in partnering with other agencies to conduct outreach activities and provide accessible services throughout the city of Atlanta. This program has brought services to communities lacking resources, extending FCDHW's reach to vulnerable populations, and addressing common transportation barriers faced by individuals in need of services. Also, interviewees noted that FCDHW's current collaboration with the correctional system, has further extended their reach and enhanced their ability to provide counseling, communicate prevention messages, and discuss linkage to care 
opportunities with inmates awaiting release. Through this partnership, FCDHW is able to facilitate better continuity of care for HIV, STD, and TB services following incarceration. Table 2 provides a historical assessment of the elements of FCDHW's previous and current approach to program collaboration, and the critical success factors and impact of their current efforts.

\section{Creating Efficiencies through Service Integration \\ "Now HIV-infected patients can be treated in either clinic for HIV, STDs and TB and can start treatment the same day." \\ -Program administrator}

Service integration was central to minimizing duplication of services and increasing efficiencies in service delivery. Administrators noted that prior to 1999, separate TB, STD, and HIV programs made it difficult for clients with co-morbid conditions to easily access needed services. Patients with multiple conditions were subject to separate registration or intake procedures for each condition, and would be referred to separate clinic locations for screening and treatment services in other disease areas. For example, an HIV-infected person visiting the HIV primary care clinic would be referred to the STD clinic for STD screening and treatment services, subjecting them to additional wait times, assessment procedures, and scheduling of clinic visits. Currently, all of these services are integrated within the HIV primary care setting.

In addition to currently integrated HIV, STD, and TB services, staff noted that numerous specialized medical and support services are also available to patients onsite. Mental health and substance abuse specialists are available to counsel patients as needed, and case managers are onsite to ensure access to housing, employment, transportation, and other needed assistance. Program administrators also reported that FCDHW has made significant efforts to incorporate the medical home model of care into its service delivery system to increase efficiencies (ACA, 2010). This involved instituting a policy ensuring that one client is paired with one primary doctor throughout the course of their care, to maintain continuity of care and better coordination of multiple services received by clients. See Table 3 for example quotes corresponding to the emerging themes.

\section{Comparison to CDC's PCSI Model}

We compared FCDHW's program to CDC's PCSI model. Because of its current delivery system, FCDHW's program best represents level three of CDC's integration model due to its array of co-located services, including onsite:1) HIV, STD, and TB screening and treatment; 2) mental health, psychosocial, and support groups; 3) substance abuse treatment; 4) case management; 5) health education and risk reduction counseling; and 6) dental services. The services provided by FCDHW extend beyond the core integrated services that CDC recommends for level 2 programs, such as HIV testing, chlamydia screening, and the provision of hepatitis B vaccines in clinical, non-clinical, and correctional settings. FCDHW staff work to ensure that individuals seen in the TB and STD clinics are able to access needed HIV prevention services such as HIV testing and risk-reduction counseling onsite. Likewise, individuals visiting the HIV primary care clinic can access STD and TB treatment and prevention services, eliminating the need for additional scheduling of appointments, and reducing the chance of loss during referral.

The availability of a variety of services in one clinic location reduces wait times, the need for separate intake procedures, and also allows for better tracking and coordination of patient care (CDC, 2009). Many of these services can be accessed the same day, enhancing the ability of individuals to access multiple services during one clinic visit without having to address transportation or other barriers to returning to the facility for additional visits.

Table 4 provides a historical assessment of the elements of FCDHW's previous and current approach to service integration, and the critical success factors and impact of its current efforts 
Table 2.

Fulton County's Current Use of Program Collaboration to Address the Needs of HIV-infected Patients, Compared to Efforts Prior to the Program's Restructure in 1999.

\begin{tabular}{|c|c|c|c|}
\hline Element & $\begin{array}{l}\text { Previous Approach } \\
\text { (Before 1999) }\end{array}$ & Current Approach & Critical Success Factors \& Impact \\
\hline Administrators & $\begin{array}{l}\text { Separate administrators } \\
\text { overseeing core } \\
\text { services for each } \\
\text { disease area (HIV, } \\
\text { STD, and TB) }\end{array}$ & - $\quad$ One administrator overseeing all three programs & $\begin{array}{l}\text { Critical success factors: } \\
\text { - } \quad \text { Consolidation of management officials } \\
\text { - } \quad \text { Facilitation of shared goals and vision } \\
\text { Impact: } \\
\text { - } \quad \text { Increased synergy across programs }\end{array}$ \\
\hline Personnel & 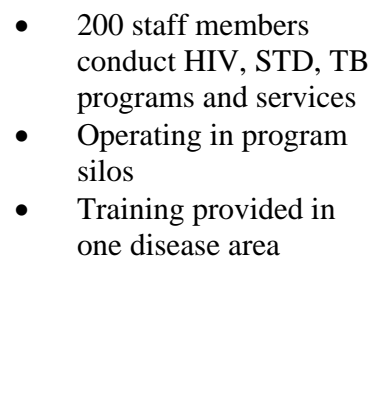 & $\begin{array}{l}\text { - } 103 \text { staff members conducting activities in an integrated } \\
\text { program with the capacity to work across different disease } \\
\text { areas }\end{array}$ & $\begin{array}{l}\text { Critical success factors: } \\
\text { - } \quad \text { Establish standard operating procedures } \\
\text { - } \quad \text { Continually assess staff strengths and abilities } \\
\text { - } \quad \text { Cross-train all staff in screening, counseling, and } \\
\text { evidence-based interventions for HIV, STD, and TB } \\
\text { - } \quad \text { Regularly communicate with staff about program } \\
\text { developments and changes (e.g., transparency) } \\
\text { Impact: } \\
\text { - } \quad \text { Increased knowledge base of staff } \\
\text { - } \quad \text { Increased flexibility to use staff across programs to fill } \\
\quad \text { service gaps }\end{array}$ \\
\hline $\begin{array}{l}\text { Community } \\
\text { partnerships }\end{array}$ & $\begin{array}{l}\text { Worked in silos - few } \\
\text { community } \\
\text { partnerships }\end{array}$ & $\begin{array}{l}\text { - Advisory group (including community members) help } \\
\text { identify program priorities } \\
\text { - } \quad \text { Partner with community-based organizations and local AIDS } \\
\text { service organizations to conduct prevention activities } \\
\text { - } \quad \begin{array}{l}\text { Partner with local organizations to provide case management } \\
\text { services }\end{array} \\
\text { - } \begin{array}{l}\text { Partner with community-based organizations and federally } \\
\text { qualified health centers to conduct screenings in high } \\
\text { morbidity areas (e.g., mobile units) }\end{array}\end{array}$ & $\begin{array}{l}\text { Critical success factors: } \\
\text { - } \quad \text { Identify and establish key partnerships in the county } \\
\text { Establish and adhere to memoranda of agreements with } \\
\text { local community-based organizations, substance abuse } \\
\text { treatment centers, and other AIDS service organizations } \\
\text { to coordinate efforts } \\
\text { Impact: } \\
\text { - Enhanced ability to provide comprehensive services }\end{array}$ \\
\hline Data systems & $\begin{array}{l}\text { - Data were collected } \\
\text { and managed } \\
\text { separately by each } \\
\text { program }\end{array}$ & $\begin{array}{l}\text { - Currently use electronic system alerting staff across HIV, } \\
\text { STD, and TB clinics of patient care needs (e.g., alert staff of } \\
\text { patient seen in HIV clinic that missed TB appointment) }\end{array}$ & $\begin{array}{l}\text { Critical success factor: } \\
\text { - } \quad \text { Develop an integrated electronic medical record system to } \\
\text { monitor multiple health outcomes (i.e., HIV, STD, TB) } \\
\text { Impact: } \\
\text { - Increased ability to monitor individual health } \\
\text { - Increased coordination of services }\end{array}$ \\
\hline $\begin{array}{l}\text { Community } \\
\text { reach }\end{array}$ & $\begin{array}{l}\text { - Limited use of } \\
\text { innovative approaches } \\
\text { to communicate } \\
\text { prevention messages }\end{array}$ & $\begin{array}{l}\text { - } \quad \text { Communicate prevention messages at non-traditional } \\
\text { nonclinical sites (e.g., colleges, jails) } \\
\text { - Communicate HIV and STD prevention messages using } \\
\text { social media (e.g., counseling prisoners via Skype ) } \\
\text { - Conduct community HIV/STD testing and outreach in high } \\
\text { morbidity areas (e.g., mobile clinics) }\end{array}$ & $\begin{array}{l}\text { Critical success factor: } \\
\text { - Identify areas where high risk persons congregate/ } \\
\text { network to communicate and provide needed services } \\
\text { Impact: } \\
\text { - Enhanced ability to access hard-to-reach clients }\end{array}$ \\
\hline
\end{tabular}


Table 3.

Emergent Themes from the Semi-structured Interviews

\section{Emergent Themes}

A holistic approach to HIV treatment

Leveraging resources through program collaboration

\section{Creating efficiencies through service} integration

\section{Examples of Participant Quotes}

“16 years ago programs were in silos with separate administrators.”(Program administrator)

"Each of the three programs had their own surveillance systems that didn't talk to each other. Now, we are working to pull all data together from HIV, STD, and TB services through one comprehensive system.” (Data manager)

"We have pharmacists on staff, and we're working to identify linkage coordinators and patient navigators to ensure clients access all the services they need.” (Program staff)

“61\% of our patients are co-infected.” (Program administrator)

“About $37 \%$ of our clients with HIV have a mental illness and about $40 \%$ have a drug problem.” (Program administrator)

"In the HIV primary care clinic, patients get a chance to get mental health and substance abuse services onsite. We also have a MOA with seven substance abuse programs in the Atlanta area.” (Program administrator)

"We contract with dental services and case managers onsite to refer clients to housing, financial assistance and nutrition services.” (Program administrator)

"We work with health centers to do education, and work with colleges where we get requests to go out and educate. We actually have a HIV clinic at Morehouse college.”(Program staff)

“We even do testing and education in jails. Now, the county’s doing Skype with jail inmates--A camera's in the exam room to interview patients before they're released. This helps connect them to care outside.” (Program administrator)

"We realized that $34 \%$ of our HIV infected clients were not keeping their appointments so we needed to come up with a way to make accessing services easier.” (Program staff)

"Having standard operating procedures in place was so important so that everybody was on the same page.” (Program staff)

"Now, HIV infected patients can be treated in either clinic for HIV, STDs and TB, and can even start treatment the same day." (Program administrator)

“Initially there was 200 staff operating in 3 silos. Now we’ve downsized to 103 staff in the integrated program.” (Program administrator)

“One big challenge was our data systems and electronic health records. We're working to consolidate data collection efforts across all of our programs.”(Data manager) 
Table 4.

Fulton County’s Current Use of Service Integration to Address the Needs of HIV-infected Patients, Compared to Efforts Prior to the Program's Restructure in 1999.

\begin{tabular}{|c|c|c|c|}
\hline Element & $\begin{array}{l}\text { Previous Approach (Before } \\
\text { 1999) }\end{array}$ & Current Approach & Critical Success Factors \& Impact \\
\hline $\begin{array}{l}\text { Services } \\
\text { available }\end{array}$ & $\begin{array}{l}\text { Level one: nonintegrated } \\
\text { services. } \\
\text { - } \quad \text { Prevention services were } \\
\text { completely separate at } \\
\text { point of client care (e.g., } \\
\text { single point testing and } \\
\text { treatment for HIV only, } \\
\text { STDs only, TB only) }\end{array}$ & $\begin{array}{l}\text { Level three: expanded integrated services } \\
\text { - } \quad \text { Comprehensive screening and treatment for HIV, STD, TB } \\
\text { - } \quad \text { Partner services (including contact investigation and } \\
\text { notification) for HIV, STD, and TB } \\
\text { - Comprehensive sexual and reproductive health services } \\
\text { (including partner services and pregnancy testing) } \\
\text { - Comprehensive risk assessment for specialized or social } \\
\text { services (i.e., case management, housing, mental health } \\
\text { services, substance abuse services etc.,) } \\
\text { - Health education services and support groups: } \\
\text { o One-on-one information to patients and their family } \\
\text { o Nutrition and HIV } \\
\text { o Risk reduction } \\
\text { o HIV education } \\
\text { o Substance abuse } \\
\text { o Adherence to therapy } \\
\text { Referrals for other services (e.g., basic dental services, weight } \\
\text { management, Medicaid referral, immunizations, aging/senior } \\
\text { services, finding employment) }\end{array}$ & $\begin{array}{l}\text { Critical success factors: } \\
\text { - } \quad \text { Cross-training of staff } \\
\text { - } \quad \text { Partnerships with organizations that provide } \\
\text { other services (i.e., substance abuse treatment } \\
\text { centers) } \\
\text { - } \quad \text { Co-located care and services } \\
\text { Impact: } \\
\text { - Increased availability and provision of } \\
\quad \text { comprehensive services }\end{array}$ \\
\hline $\begin{array}{l}\text { Service delivery } \\
\text { system }\end{array}$ & $\begin{array}{l}\text { - Clients with comorbidities } \\
\text { had to visit multiple } \\
\text { clinics for care and } \\
\text { treatment } \\
\text { - } \quad \text { Increased wait times } \\
\text { - Duplicative clinical } \\
\text { assessment for client with } \\
\text { co-morbidities seen at } \\
\text { multiple sites }\end{array}$ & $\begin{array}{l}\text { - } \\
\text { - } \quad \text { lo care } \\
\text { - Conduct comprehensive screening (HIV, STD, TB) and risk- } \\
\text { reduction counseling at all clinics } \\
\text { - } \quad \text { Decreased wait times } \\
\text { - } \quad \text { Reduced need for separate intake procedures } \\
\text { Better tracking and coordination of patient care }\end{array}$ & $\begin{array}{l}\text { Critical success factors: } \\
\text { - On-site laboratory and pharmacy allows for } \\
\text { - } \quad \text { Extensive cross-training for all staff in } \\
\text { screening and counseling for HIV, STD, and } \\
\text { TB services } \\
\text { - } \text { Additional regular staff training as evidence- } \\
\text { based interventions are added } \\
\text { - Collect patient satisfaction surveys which } \\
\text { lead to improved service delivery } \\
\text { Impact: } \\
\text { - Increased efficiency in service delivery }\end{array}$ \\
\hline $\begin{array}{l}\text { Linkage to care } \\
\text { and other } \\
\text { services }\end{array}$ & $\begin{array}{l}\text { - Limited linkage } \\
\text { coordinators or staff } \\
\text { available }\end{array}$ & $\begin{array}{l}\text { - Case managers available onsite to assist patients with complex } \\
\text { social and medical needs (i.e., housing, employment, } \\
\text { transportation) } \\
\text { - Currently establishing a system utilizing linkage coordinators } \\
\text { and patient navigators to facilitate patient access to services. }\end{array}$ & $\begin{array}{l}\text { Critical success factor: } \\
\text { - Co-located services within HIV, STD, and TB } \\
\text { clinics allow for easy access to services. } \\
\text { Impact: } \\
\text { - Increased linkage to care and referral to other } \\
\quad \text { services }\end{array}$ \\
\hline
\end{tabular}




\section{Content Analysis Results}

We identified 17 programmatic documents providing evidence of PCSI efforts. The analysis of these documents corroborated key findings from the interviews. Internal documents, including the integrated care standard operating procedures, organizational charts and referral flow charts showed a shift in available services in 1999. Documents showed that FCDHW expanded its collaborations to leverage community resources (i.e., staff and funds), and enhanced its capacity to respond comprehensively to multiple patient needs. Collaborations with community-based organizations and relationships built through the mobile van program became increasingly apparent in communication materials, such as flyers, pamphlets, and resource documents highlighting FCDHW's available services. Evidence was further apparent in internal documents establishing memoranda of agreements (MOAs) with seven substance abuse agencies throughout the Atlanta metropolitan area to provide appropriate referrals to in-patient treatment programs for patients requiring longterm, intensive, substance abuse services. This was further displayed through program guidance documents describing appropriate referral protocols and procedures for these services.

Clinic flow diagrams also showed that FCDHW enhanced service integration to minimize duplication of services and increase efficiencies in service delivery. These charts displayed the recommended flow of clients with co-morbid conditions through the HIV primary care clinic, indicating areas of integrated service provision. Specifically, documents showed areas where STD and TB services are currently delivered onsite in the HIV primary care clinic, limiting the need for referral to STD or TB clinics and reducing the potential for loss to follow up.

\section{Discussion}

This case study contributes to the current literature by highlighting specific strategies local health departments can use to enhance the provision of comprehensive services to HIVinfected clients. There are few case studies in the literature showcasing practice-based strategies public health organizations can implement to enhance collaboration and integration of services. This study provides specific approaches to establishing a delivery system where patients can access needed services efficiently, with reduced administrative barriers and enhanced coordination of care. It also showcases the value of collaborating with an array of community entities to leverage resources to address the diverse needs of HIVinfected clients. When compared to CDC's PCSI model, FCDHW's program represents a high level of integration due to the availability of numerous co-located services, extending beyond the core level of services recommended by CDC.

This case study also identified key lessons learned, highlighting major facilitators and challenges to successful PCSI implementation. The importance of: a) establishing clear goals and objectives for implementing PCSI efforts, b) establishing clear and consistent standard operating procedures (SOPs) to guide program efforts, c) incorporating ongoing cross-training of staff in multiple disease areas (i.e., HIV, STD, TB); d) conducting ongoing assessments of staff strengths and abilities, and e) gaining continuous feedback and buy-in from staff during the restructure, was made clear through interviewees' discussion of facilitators of PCSI implementation.

Some of the challenges noted were: a) staff resistance to change, b) extensive time required to cross-train staff and develop detailed operational procedures, and c) the lack of adequate space for the integrated program. FCDHW continues to work to address ongoing implementation issues, such as integrating surveillance systems, identifying additional space to provide comprehensive services, and balancing incoming referrals from CBOs.

\section{Limitations}

The limitations of this case study include our inability to conduct a comprehensive evaluation of the program due to resource constraints, thereby, limiting our capacity to comment on program effectiveness. Also, the results were 
primarily based on program administrator feedback, clinic observations, and review of program planning and communications documents. Thus, there was limited analysis of resources external to the program to corroborate evidence.

Also, while confounding factors were not assessed, factors that potentially affected the study include: recall bias, policy changes over time that impacted the program, programmatic restructuring and priorities, fluctuations in funding resources, and changing organizational leadership staff. The findings of this case study are not generalizable to other programs due to these limitations; however they serve as lessons learned for programs considering implementation of PCSI.

\section{Conclusions}

The FCDHW serves as an example of enhanced program collaboration and service integration within a local public health department setting. FCDHW's experience, including the lessons learned and challenges encountered, can inform the efforts of similar county or state level health departments contemplating incorporating PCSI into their current efforts. This example is particularly relevant to public health systems serving high HIV prevalence areas, and systems with large numbers of HIV-infected clients with complex medical and support needs.

Future studies exploring the role of collaboration and service integration within a local public health department setting could compare two or more different models of collaboration and service integration within a community and describe perceived benefits and burdens from the patient and the service providers' perspective. Depending on the needs of the local community seeking care and treatment from the local health department, different types of nonHIV related services may have varying levels of benefits observed and/or burden imposed on the service provider as well as the patients.

\section{Acknowledgements}

The authors would like to acknowledge the contributions of Veronica Hartwell and Ruby Lewis-Hardy of the Fulton County Department of Health and Wellness. They were instrumental in providing key historical insight into the county's program collaboration and integration efforts. The findings and conclusions in this report are those of the authors and do not represent the views of the Centers for Disease Control and Prevention (CDC), the National Institutes of Health(NIH), or the Fulton County Department of Health and Wellness. At the time this article was developed, Dr. Patricia L. Jones was employed with the Division of HIV/AIDS Prevention at the CDC; she is currently employed with the National Institutes of Health (NIH). At the time this article was developed Dr. Stephanie J. Bernard was employed with the Division of HIV/AIDS Prevention at the CDC; she is currently employed with the Division for Heart Disease and Stroke Prevention at the CDC.

\section{References}

ACA. (2010). Patient Protection and Affordable Care Act, Public Law 111-148, 124 Stat., 119. Retrieved from http://www.gpo.gov/fdsys/pkg/PLAW-111publ148/pdf/PLAW-111publ148.pdf.

Aidala, A.,Wilson, M., Shubert,V., Gogolishvili, D., Globerman, J., Rueda, S., Bozack, A., Caban, M., and Rourke, S. (2015). Housing Status, Medical Care, and Health Outcomes Among People Living With HIV/AIDS: A Systematic Review. American Journal of Public Health, 106(1), 9595.

Aidala, A., Lee, G., Abramson, A., Messeri, P., and Siegel, A. (2007). Housing need, housing assistance, and connection to HIV medical care. AIDS and Behavior, 11, S172-S181.

Altice, F.L., Kamarulzaman, A., Soriano, V.V., Schechter, M., Friedland, G.H. (2010). Treatment of medical, psychiatric, and substance-use comorbidities in people infected with HIV who use drugs. Lancet, 376(9738), 367-87.

Carter GM, Indyk D, Johnson M, Andreae M, Suslov K, Busani S, et al. (2015). Micronutrients in HIV: A Bayesian Meta-Analysis. PLoS ONE 10(4). 
Centers for Disease Control and Prevention. (2009). Program Collaboration and Service Integration: Enhancing the Prevention and Control of HIV/AIDS, Viral Hepatitis, Sexually Transmitted Diseases, and Tuberculosis in the United States. Atlanta, GA: US Department of Health and Human Services, Centers for Disease Control and Prevention, 1:1-45. Retrieved from http://www.cdc.gov/nchhstp/programintegration/docs/207181-

C_NCHHSTP_PCSI\%20WhitePaper-508c.pdf

Centers for Disease Control and Prevention. (2012). Integrated prevention services for HIV infection, viral hepatitis, sexually transmitted diseases, and tuberculosis for persons who use drugs illicitly: Summary guidance from CDC and the U.S. Department of Health and Human Services. Mortality and Morbidity Weekly Report, Recommendations and Reports, 61(5), 1-47. Retrieved from http://www.cdc.gov/mmwr/preview/mmwrhtml/rr6105a1.htm?s_cid=rr6105a1_w

Cole, S.W. (2008). Psychosocial influences on HIV-1 disease progression: Neural, endocrine, and virologic mechanisms. Psychomatic Medicine, 70, 562-68.

Evans, K.R. (2006). Ancillary services for individuals living with HIV/AIDS. Nursing Clinics of North America, 383-93.

DHHS, Center for Medicaid, CHIP and Survey \& Certification. (2010). Health homes for enrollees with chronic conditions. Retrieved from http://www.healthreformgps.org/wpcontent/uploads/he11172010_HealthHome.pdf.

Fulton County Department of Health and Wellness, Communicable Disease Prevention Branch. (2012). Monthly report, June 2012.

Gallant, J.E., Adimora, A.A., Carmichael, K.J., Horberg, M., Kitahata, M., Quinlivan, E.B., Raper, J.L., Selwyn, P., Williams, S.B. (2011). Essential components of effective HIV care: A policy paper of the HIV Medicine Association of the Infectious Diseases Society of America and the Ryan White Medical Providers Coalition. Clinical Infectious Diseases, 53(11), 1043-50.

Ickovics, J.R., Milan, S., Boland, R., Schoenbaum,E., Schuman, P., Vlahov, D. (2006). Psychological resources protect health: 5-year survival and immune function among HIV-infected women from four US cities. AIDS, 20, 1851-60.

Institute of Medicine. (2011). HIV screening and access to care: Exploring the impact of policies on access to and provision of HIV care. Washington, DC: The National Academies Press. Retrieved from http://iom.edu/Reports/2011/HIV-Screening-and-Access-to-Care-Exploring-the-Impact-ofPolicies-on-Access-to-and-Provision-of-HIV-Care.aspx.

Ironson, G.H., and Hayward, H. (2008). Do positive psychosocial factors predict disease progression in HIV-1? A review of the evidence. Psychosomatic Medicine, 70(5), 546-554.

Leaver ,C.A., Bargh, G., Dunn, J.R., Hwang, S.W. (2007). The effects of housing status on health-related outcomes in people living with HIV: A systematic review of the literature. AIDS Behavior, 11, S85-S100.

Lee, W. K., Milloy, M. J. S., Walsh, J., Nguyen, P., Wood, E., \& Kerr, T. (2015). Psychosocial Factors in Adherence to Antiretroviral Therapy Among HIV-Positive People Who Use Drugs. Health Psychology. Advance online publication. http://dx.doi.org/10.1037/hea0000310

Leserman, J. (2008). Role of depression, stress, and trauma in HIV disease progression. Psychomatic Medicine, 70, 539-545.

Mahlungulu, S., Grobler, L.A., Visser, M.E., Volmink, J. (2007). Nutritional interventions for reducing morbidity and mortality in people with HIV. Retrieved from the Cochrane Database of Systematic Reviews. (CD004536)

O’Brien, K., Nixon, S., Tynan, A.M., Glazier, R. (2010). Aerobic exercise interventions for adults living with HIV/AIDS. Retrieved from the Cochrane Database of Systematic Reviews. (CD001796)

Nixon, S., O'Brien, K., Glazier, R.H., Tynan, A.M. (2005). Aerobic exercise interventions for adults living with HIV/AIDS. Retrieved from the Cochrane Database of Systematic Reviews. (CD001796)

Riley, E., Gandhi, M., Hare, C.B., Cohen, J., Hwang, S. (2007). Poverty, unstable housing, and HIV infection among women living in the United States. Current HIV/AIDS Reports, 4, 181-186. 
Sackoff, J.E., Hanna, D.B., Pfeiffer, M.R., Torian, L.V. (2006). Causes of death among persons with AIDS in the era of highly active antiretroviral therapy: New York City. Annals of Internal Medicine, 145(6), 397-406.

Shubert, V., Bernstine, N. (2007). Moving from fact to policy: Housing is HIV prevention and health care. AIDS Behavior, 11, S172-S181.

Spencer, L.M., Schooley, M.S., Anderson, L.A., Kochtitzky, C.S., DeGroff, A.S., Devlin, H.M., Mercer, S.L. (2013). Seeking best practices: A conceptual framework for planning and improving evidencebased practices. Preventing Chronic Disease, 10:130186.

Swendeman, D., Ingram, B.L., and Rotheram-Borus, M.J. (2009). Common elements in self-management of HIV and other chronic illnesses: an integrative framework. AIDS Care, 21(10), 1321-1334.

Tambe, P., Allen, M., Hardy, R.L., Dupree-Bright, T., Benning, E., Katkowsky, S. (2008). Effect of clinical program integration on eliminating disparities in access to care. Presentation at the 2008 National STD Prevention Conference.

White, J.M., Gordon, J.R., and Mimiaga, M.J. (2014). The Role of Substance Use and Mental Health Problems in Medication Adherence Among HIV-Infected MSM. LGBT Health, 1(4): 319-322.

World Health Organization. (2003). Who Technical Consultation on Nutrient Requirements for People Living with HIV/AIDS. Geneva: World Health Organization.

Zahari, M.M., Hwan Bae, W., Zainal, N.Z., Habil, H., Kamarulzaman, A., Altice, F.L. (2010). Psychiatric and substance abuse comorbidity among HIV seropositive and HIV seronegative prisoners in Malaysia. American Journal of Drug and Alcohol Abuse, 36(1), 31-38.

Zaller, N., Gillani, F.S., Rich, J.D. (2007). A model of integrated primary care for HIV-positive patients with underlying substance use and mental illness. AIDS Care, 19(9), 1128-33.

Author Information

Stephanie J. Bernard, Ph.D., M.P.H.

Division for Heart Disease and Stroke Prevention

National Center for Chronic Disease Prevention and Health

Promotion

Centers for Disease Control and Prevention

Telephone: 770-488-5108

Fax: 770-488-8151

E-mail: sbernard@cdc.gov

* corresponding author 